3D landmarks of Craniofacial Imaging and subsequent Considerations on Superimpositions in Orthodontics - The Aarhus Per spective

Running title:

3D landmarks in Orthodontics

Paolo M. Cattaneo ${ }^{1}$

Augustine. K.C. Yung ${ }^{1}$

Annemarie Holm ${ }^{1}$

Omar M. Mashaly ${ }^{1}$

Marie A. Cornelis ${ }^{1}$

1) Section of Orthodontics, Department of Dentistry and Oral Health, Aarhus University, Aarhus, Denmark

Correspondence to:

DrPaolo M. Cattaneo

Department of

\title{
Orthodontics
}

I nis is the autnor manuscript acceptea tor publication ana nas undergone tulı peer review but has not been through the copyediting, typesetting, pagination and proofreading process, which may lead to differences between this version and the Version of Record. Please cite this article as doi: 10.1111/OCR.12299

This article is protected by copyright. All rights reserved 


\section{School of Dentistry}

University of Aarhus

Vénnelyst Boulevard

9

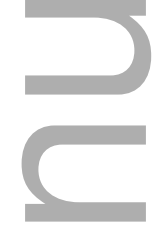

层

DK-8000 Aarhus

Dènmark

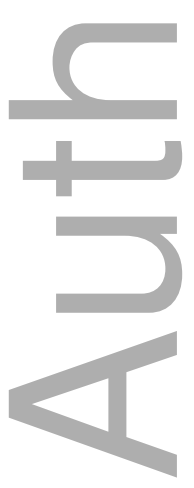




\section{E-mail:}

pälo.cattaneo@odon

tologi.au.dk

DrPaolo M. Cattaneo

Department

of

\section{Orthodontics}

Sehool of Dentistry

University of Aarhus 


\section{Vennelyst Boulevard}

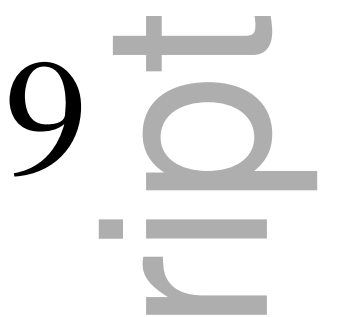

DK-8000 Aarhus

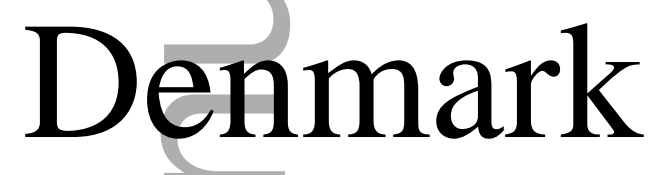

E-mail:

paglo.cattaneo@odon

tộgi.au.dk

Paolo M. Cattaneo

Section of Orthodontics, Department of Dentistry and Oral Health, Aarhus University

Vennelyst Boulevard 9

DK-8000 Aarhus, DENMARK

E-mail:paolo.cattaneo@dent.au.dk 
Keywords: CBCT, 3D, Cephalometry, Superimposition, Orthodontics

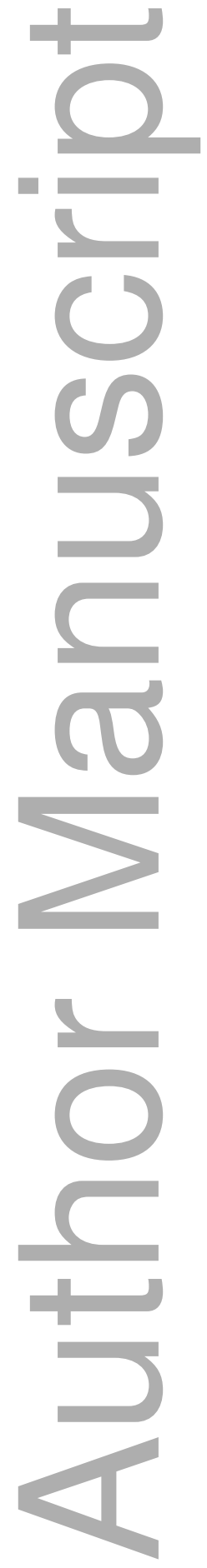




\section{Article Type: Supplement Article}

\section{ABSTRACT}

Objective: 1. to evaluate intra- and inter-observer reliability in landmarks placement along the three planes of space on CBCT data sets; 2. to evaluate whether the reliability of each landmark differs in CBCT scans characterized by two different voxel dimension and quality.

Setting and Sample Population: A total of 84 scans were used in this study: 49 scans were taken with the NewTom $3 \mathrm{G}$ and 35 scans were taken with the NewTom 5G. The scans were characterized by an isotropic voxel dimension of $0.36 \mathrm{~mm}$ and $30 \mathrm{~mm}$ for the NewTom $3 \mathrm{G}$ and the NewTom $5 \mathrm{G}$ respectively.

Methods: A total of 13 landmarks were placed according to the corresponding definitions in 3D, also presented in this study: Foramen Spinosum (R/L), Nasion, Sella, Gonion (R/L), Pogonion, Menton, A point, Anterior nasal spine, Posterior nasal spine, Basion, Cribriform Plate (CR). Intra- and inter-observer reliability and ICC for landmarks identification were assessed. Five reference and registration planes based on the above-mentioned landmarks, were also presented.

Results: ICC both for the intra- and inter-observer measurements had a score larger than 0.9 in all directions, except in the sagittal direction for CR. Regarding intra- and inter-observer reliability, only $\mathrm{N}, \mathrm{S}$, and Ba scored well in all directions.

Conclusions: most of the landmarks analyzed displayed a high reliability along at least 2 directions. The choice of landmarks to define registration and superimposition planes must be carefully selected, as the reliability of these planes is inherited from the one of the landmarks defining them.

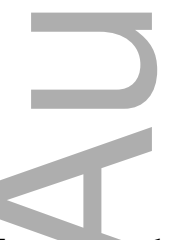

Keywords: cone-beam computed tomographic; orthodontics; three-dimensional imaging; cephalometry; superimposition. 


\section{Introduction}

Conventional cephalometric analysis, which has been used for almost a century, is performed by identifying landmarks on two-dimensional (2D) images. ${ }^{1}$ Although the magnitude of the errors when identifying the craniofacial structures (landmarks) on 2D images have been previously addressed, ${ }^{2,3}$ cephalometry has been and still is a valuable method to diagnose and evaluate growth and treatment outcomes of orthodontic patients.

Computed tomography (CT) has been used to depict the three dimensional (3D) morphology of the cranium since 1971, but its application in dentistry has been limited to selected patients, due to high levels of radiation, availability, and scanning costs. Following the technological advancements seen in dentistry in the last years mostly due to the introduction of CBCT scanners and 3D facial camera, it has become possible to represent threedimensionally the structures of the maxillofacial complex for a larger population, yet keeping the ionizing radiation dose to the patients to a minimum (CBCT) or without radiation (3D facial camera). This allows orthodontists to visualize craniofacial structures in $3 \mathrm{D}$, thus overcoming the limitations of $2 \mathrm{D}$ cephalometric analysis, without the drawbacks of CT scanning.

During the transition from 2D to 3D radiographic data sets, some researchers compared linear and angular measurements performed on CBCT-synthesized cephalograms to the equivalent measurements taken on conventional cephalometric images, concluding that the measurements are similarly accurate and therefore comparable. ${ }^{6-8}$ Yet, this approach still represents a $2 \mathrm{D}$ methodology, and, consequently, these results cannot be considered useable for validating 3D cephalometric analyses.

The next step followed by some researchers towards a 3D analysis was to measure the maxillofacial skeleton directly on the three planes of space, still borrowing some of the methods from conventional cephalometric analysis. Most of the well-established 2D landmarks definitions were conceived having in mind the sagittal plane view (i.e. along the vertical or antero-posterior direction). Whether the 2D-based norm values are valid in 3D has not been fully elucidated. ${ }^{4,9}$ It is implicit, that to developing a truly $3 \mathrm{D}$ cephalometric analysis requires identification of landmarks on complex curved structures along the three planes of space. For this reason, some of the definitions of the landmarks used in 2D need to be updated (e.g. Porion); ${ }^{10}$ while some 2D landmarks cannot be used, as they simply do not exist in $3 \mathrm{D}$ (e.g. Articulare) as they are the result of $2 \mathrm{D}$ projections of structures laying on 
different planes. ${ }^{11}$ Moreover, different structures are characterized by different features so that placing a landmark at the central part of a concave structure (e.g. Sella Turcica) is quite different from placing a landmark on an area of maximum curvature (e.g. Gonion), or at the outermost point of a projection (e.g. Anterior Nasal Spine).

Due to the complexity of some anatomical structures in 3D, errors in landmark identification represent a major source of cephalometric errors. ${ }^{12}$ Therefore, comprehensive definitions of landmarks along all three directions (i.e. sagittal, vertical, and transversal) are crucial to decrease the uncertainties in landmark identification. As done previously for 2D cephalometric analyses, it is essential to develop and validate standardized protocols to define and identify novel landmarks in 3D. ${ }^{6,10,11,13,14}$ Trpkova and co-workers made a ranking of the most reliable 2D landmarks along the sagittal and vertical directions in their systematic review. ${ }^{15}$ The same ranking attempt was done by some authors for $3 \mathrm{D}$ landmark identification, ${ }^{16,17}$ yet, it has also been advocated that a standardization of landmark selection is still needed. ${ }^{4,18}$

The accuracy and reliability of linear measurements on CBCTs represent other aspects that have been investigated. ${ }^{19-21}$ Although these studies generally presented good results, linear measurements are not useful to determine the accuracy of landmark identification: linear measurements involve two points, thus rendering it impossible to determine the accuracy of each single landmark. ${ }^{9}$ This side effect is exacerbated by the fact that large variations (e.g. a variety of CBCT scanners and different scanning parameters were used; lack of inter-rater assessment, etc.) are present between the different studies.

Partial volume effect and reconstruction artifacts may result in blurry or irregular images, thus resulting in substantial errors in the images, especially when the voxel dimension is large. $^{22}$ As a result, the quality of the CBCT scans may affect the reliability of landmark identification, yet this is a matter that was not clearly assessed previously before and so needs to be further investigated.

In order to have reliable measurements depicting craniofacial morphology, reference planes must be correctly defined: landmarks are commonly defined along directions, and thus their placement may vary in relation to the definition of the reference planes. ${ }^{23}$

In orthodontics, in order to assess growth, treatment effects and/or long-term stability, registration of two or more images is usually performed. Historically, registration was performed on plain cephalograms, although the superimposition was shown to be 
challenging. The midsagittal plane (MSP) and the Frankfort Horizontal (FH) plane are two of the planes commonly used to perform registration (or superimposition) of cephalograms taken at different time points are. ${ }^{24}$ Some issues have been raised for the definitions of both planes: for the MSP, it was questioned whether a true craniofacial symmetry exists; ${ }^{25}$ for the $\mathrm{FH}$, whether this plane can be determined in a reproducible way. ${ }^{26}$

In order to unravel the above-mentioned problems, there are three challenges that needs to be solves. The first challenge is to determine which structures /landmarks are more suitable to be used for registration.

The way the registration is performed represents the second challenge. At present, there are mainly three methods used to superimpose 3D images: 1) based on landmarks; 2) based on surfaces; and 3) based on voxels. In order to produce reliable results, all these methods should be relying on stable anatomical structures, ${ }^{27,28}$ and this leads to the fact that each of these superimposition methods is characterized by drawbacks: 1) the landmarks might be difficult to identify and place correctly; 2) surfaces are depending on the chosen threshold; 3) voxel-based is generally time-consuming.

The third challenge is related to the way the results are reported after image registration. Again, several methods have been proposed: 1) absolute displacement of landmarks; 2) colorcoded images based on the closest points-distance; and 3) shape analysis, where the distances between corresponding points are calculated, are the ones used most.

The focus of the present study was to assess the reliability in identifying landmarks on CBCT data-sets and in defying the associated reference planes. This was addressed through the following aims:

1) To evaluate intra- and inter-observer reliability in placing landmarks along the three planes of space;

2) To evaluate whether the reliability of each landmark differs in CBCT scans characterized by two different voxel dimension and quality.

\section{Material and Method}

In this retrospective study, approved by the XXX Data Protection Agency (reference 201557-0002, XXXX 62908), informed consent was obtained from all patients. The CBCT scans had been taken for orthodontic diagnosis and treatment planning at the Section of 
Orthodontics, Faculty of Health Sciences, Aarhus University, therefore, none of the patients was exposed to any extra radiation for the purpose of this project. The inclusion criterion was the availability of a large field of view CBCT scan. The exclusion criteria were: 1) presence of a cleft; 2) degenerative conditions; 3) history of trauma; 4) orthognathic surgery; and 5) CBCT scans with clear motion artifacts and/or absence of maximum intercuspation during acquisition

The CBCT scans were acquired by either NewTom 3G or $5 \mathrm{G}$ machines (QR-Verona, Italy): the generated data sets are characterized by an isotropic voxel dimension of $0.36 \mathrm{~mm}$ and 30 mm for the NewTom $3 \mathrm{G}$ and the NewTom $5 \mathrm{G}$ respectively. A total of 84 scans could be identified: 49 scans were taken with the NewTom 3G, whilst 35 scans were taken with the NewTom 5G. The CBCT scans were anonymized, exported via DICOM format, and then imported into a 3D image-processing program (MIMICS 19.0, Materialise, Leuven, Belgium).

The threshold used to segment and visualize the bony structures was set individually for each CBCT scan, with particular attention given to the cranial base. Based on this threshold value, a 3D layer with the relevant structures was defined. From a mask, the corresponding craniofacial structure was generated, and could be visualized in 3D (Figure 1).

A total of 13 landmarks were placed according to the corresponding definitions (Table 1) 13,17,29,30 in Mimics software, following the procedure described by Di Carlo et al. ${ }^{31}$ All midsagittal landmarks were identified on the sagittal images and their position checked on the coronal and axial planes; then their placement was identified on the 3D surface; finally, they were adjusted by finely relocating them on the axial, coronal and sagittal views. These landmarks were chosen as they are used to generate the following reference and superimposition planes (Table I):

- Horizontal reference plane: passing through the two Foramina Spinosa and through Nasion. This is not a true horizontal plane: it is only used to make sure that the next three planes will be constructed and oriented according to the cranial base, used as reference structure.

- Sagittal plane: constructed perpendicular to the horizontal reference plane and passing through Sella and Nasion. This is a reference plane, but can also be used for registration; 
- The axial plane: defined as the plane passing through Sella and Nasion, and perpendicular to the sagittal plane. This is a reference plane, but can also be used for registration;

- Coronal plane: defined as the plane passing through Sella, and perpendicular to the axial and sagittal planes;

- Cribriform plane: defined as the plane passing through Sella and Cribriform plate mid-point, and perpendicular to the sagittal plane. This serves only as a registration plane.

Three operators (RH, AKCY, and OMM) were instructed about landmarks definition and software operation; they were then given a number of data sets for training and calibration, which they performed together with the principal investigator (PMC - with extensive expertise both with software and 3D landmark identification) until they felt confident with the procedure. One operator identified and marked all the 13 anatomic landmarks on the whole set of CBCTs and repeated the whole procedure with a three-week period interval, while the other two operators identified and marked respectively 11 and 2 landmark(s) once.

The X-, Y-, Z- coordinates (i.e. X - transverse; Y - sagittal; Z - vertical) of all the landmarks were exported to an Excel spreadsheet (Microsoft, Washington, USA).

\section{Statistical analysis}

Descriptive statistics were computed using SPSS (IBM Corp, Version 25.0. Armonk, NY). The difference in the landmark coordinates along the 3 directions $(\mathrm{X}, \mathrm{Y}, \mathrm{Z})$ were analyzed for intra- and inter-observer reliability using both the Dahlberg formula, to quantify the technical error of measurements, and the Bland Altman plot. ${ }^{32,33}$ The related limits of agreement (LoA; $1.96 * \mathrm{SD}$ ) were calculated as well. Intra-class correlation coefficients (ICC) were calculated using a two-way mixed effect model to assess intra-rater and inter-rater correlation. Dahlberg values were judged good if smaller than $0.7 \mathrm{~mm}$, LoA were judged good if smaller than 1.4 $\mathrm{mm}$ (respectively 2 and 4 times the voxel dimension), ${ }^{34}$ ICC above 0.9 was judged as very good.

\section{$\underline{\text { Result }}$}


All the results are reported in Table II. The present study showed that the ICC (for the intraand inter-observer measurements) had a score larger than 0.9 in all directions, except in the sagittal direction for the CR-m (Table II).

Regarding the intra- and inter-observer reliability both in terms of the Dahlberg and the LoA, as defined by Bland and Altman, only N, S, and Ba scored well in all directions. The other landmarks scored well in at least two directions, with the exception of Gonion, which was reliable only along the $\mathrm{x}$-transverse direction.

A large difference in respect to both the Dahlberg and LoA was seen along the Y-Sagittal direction for Cribriform Plate, Gonion, and Menton; whilst Gonion, Pogonion, and A point displayed a large variation along the Z-Vertical direction.

The differences for both Dahlberg and LoA were generally smaller in case of scanning generated by the NewTom 5G: looking at the FS, the error in measuring performed on these scans exhibited smaller values than the corresponding measurements on scans from NewTom $3 \mathrm{G}$.

In general, the calculated intra- and inter-observer differences were similar.

\section{Discussion:}

The present study focuses on the choice of reliable landmarks, which are needed to generate reference and superimposition planes, when using 3D data-sets generated from CBCTscanners.

In $2 \mathrm{D}$ cephalometry, measurements are typically made using either the distance between 2 landmarks or as linear and/or angular measurements between two lines, supposedly marking some anatomical structures as seen on the 2D (sagittal) projection. When moving to 3D cephalometry, the possibility to identify the landmarks in their actual location in the 3D space is possible. Hence, it is possible to identify not only the "classical" structures (i.e. the ones that are characterized by a clear contour in the $2 \mathrm{D}$ projection and which are not bilateral) but to look for structures and other anatomical features, which are not clearly visible on conventional plain cephalograms (e.g. foramina). Moreover, it is possible to measure distances outside the in-plane directions, and angles between planes instead as between lines are assessed. 
From the present study, it is clear that each specific landmark presents different performance in terms of reliability and accuracy along the three spatial dimensions, indicating, as previously stated for 2D analysis, that each landmark has its own "envelope of error".35 Accordingly, each landmark should be chosen and used keeping in mind its performance along the specific direction it will be used for, and for which plane it will be used. This is in agreement with what was reported in a previous research. ${ }^{18,29}$$$
\text { (1) }
$$

As an example, according to results of this study, Gonion, which displayed poor reliability along the sagittal and vertical direction, exhibited higher reliability along the $\mathrm{X}$ direction, and thus can be used for assessing transverse dimensions of the dentofacial complex. On the opposite, using Gonion to determine vertical or sagittal dimensions or in the definition of planes perpendicular to the sagittal direction would generate large errors, and thus would not be suitable to measure vertical and antero-posterior dimensions. This is in accordance with what Baumrind and Frantz stated in their classical paper: “...large differences in reliability of estimation among several landmarks..." were seen and that "Gonion and lower incisor apex are clearly the least reliable landmarks". 2 The reasons behind these uncertainties are depending on the difficulties in locating landmarks along broadly curved structures. Moreover, what became clear in the present study is that the erroneous identification of landmarks can be improved by suggesting a proper explicit definition together with a precise sequence in order to identify those landmarks on the MPR view and/or on the 3D rendering view (Table 1$)$.

The present study showed that along the vertical direction, landmark CR-m exhibited low reliability, while Nasion performed well. Consequently, coming to our third challenge when it comes to choose landmarks for superimposition, non-growing patients, or when the time difference between two consecutive scans covers a short period of growth, it might be more appropriate to use $\mathrm{N}$ instead of $\mathrm{CR}-\mathrm{m}$ for. This corroborates what was previously stated by Pancherz and Hansen, reporting that the error in registration using the anterior cranial base was larger than using the N-S line, as the inter-observer difference was larger for the anterior cranial base. ${ }^{36}$

It is known already from the 50's that Nasion is one of the landmarks affected by growth, ${ }^{37}$ and this finding were subsequently confirmed by a recent systematic review. ${ }^{28}$ Therefore, in case of growing patients the use of CR-m instead of $\mathrm{N}$ might be a better choice in order to 
minimize superimposition errors. The presphenoid and cribriform plate regions are indeed considered stable after age seven, making them the best cranial-base superimposition areas; ${ }^{28}$ these observations were previously verified histologically. ${ }^{38}$ The idea of using landmarks located on the anterior cranial base is in accordance with the findings of Baumrind and coworkers, ${ }^{39}$ stating that biological induced errors (i.e. growth modifications) are the cause of the primary errors when performing superimpositions.

Lagravere and co-workers assessed that the foramina spinosa displayed low identification error in both the vertical and horizontal directions; ${ }^{29}$ this was observed in the present study as well, with the exception of the inter-observers LoA for the vertical direction.

When comparing the results from the measurements done on scans from two CBCT-scanners, it is evident that the resolution and voxel quality of CBCT data-sets needs to be taken into consideration: indeed, the identification error, both for the intra-and inter-observer agreement, differs with respect to the two different scanners (reported in Table II). Thus, CBCT-related image quality seems to play a role, though for some landmarks only marginal. As a general rule, the present results suggest that the smaller the voxel dimension and the lesser noisy the images are, the easier and more reliable the landmarks will be located. This is particularly evident in case of complex anatomical structures (e.g. foramen spinosum).

The landmarks used for defining the planes proposed in this article were chosen both for their widespread used in the literature and because of their biologic relevance. In case of the Horizontal reference plane, both Nasion and the two FS R/L were chosen as they represent well the anterior portion of the craniofacial structure and the middle cranial fossa respectively, thus they retain the additional resulting feature to be located far apart. Indeed, the closer the landmarks are located, the greater the angular error of the plane they define; in other words, the influence of the landmarks identification is minimized when the landmarks used to define a plane are situated as far as possible from each other. The same approach was used to define the other planes. Based on the results of this study, it can also be concluded that the choice of reference planes must be supported by the accuracy and reliability of the landmarks defining them. Thus, if a plane is used as a reference for measuring dimensions along the vertical direction, the landmarks used to define such a plane must be very reliable in the vertical dimension. Therefore, despite the fact that the CR-m was shown to be difficult to identify along the sagittal direction, given the anatomic shape of the cribriform plate, it should not be seen as a hinder to use it as a reference or registration plane for the vertical aspects. 


\section{Conclusion}

Most of the landmarks considered in the present study displayed a high accuracy and reliability in at least 2 directions, however the direction of observation highly determines the reliability of each landmark.

Planes and superimposition technique are inheriting the reliability from the landmarks they are based upon: the landmarks used to define these planes must be carefully selected.

\section{References}

1. Hans MG, Palomo JM, Valiathan M. History of imaging in orthodontics from Broadbent to cone-beam computed tomography. Am J Orthod Dentofacial Orthop. 2015;148(6):914-921.

2. Baumrind S, Frantz RC. The reliability of head film measurements. 1. Landmark identification. American journal of orthodontics. 1971;60(2):111-127.

3. Bjork A, Solow B. Measurement on radiographs. J Dent Res. 1962;41:672-683.

4. Pittayapat P, Limchaichana-Bolstad N, Willems G, Jacobs R. Three-dimensional cephalometric analysis in orthodontics: a systematic review. Orthod Craniofac Res. 2014;17(2):69-91.

5. Cattaneo PM, Melsen B. The use of cone-beam computed tomography in an orthodontic department in between research and daily clinic. World journal of orthodontics. 2008;9(3):269-282.

6. van Vlijmen OJ, Berge SJ, Swennen GR, Bronkhorst EM, Katsaros C, KuijpersJagtman AM. Comparison of cephalometric radiographs obtained from cone-beam computed tomography scans and conventional radiographs. J Oral Maxillofac Surg. 2009;67(1):92-97.

7. Kumar V, Ludlow JB, Mol A, Cevidanes L. Comparison of conventional and cone beam CT synthesized cephalograms. Dento maxillo facial radiology. 2007;36(5):263269.

8. Cattaneo PM, Bloch CB, Calmar D, Hjortshoj M, Melsen B. Comparison between conventional and cone-beam computed tomography-generated cephalograms. Am J Orthod Dentofacial Orthop. 2008;134(6):798-802. 
9. Fuyamada $\mathrm{M}$, Shibata $\mathrm{M}, \mathrm{Nawa} \mathrm{H}$, et al. Reproducibility of maxillofacial landmark identification on three-dimensional cone-beam computed tomography images of patients with mandibular prognathism: Comparative study of a tentative method and traditional cephalometric analysis. Angle Orthod. 2014;84(6):966-973.

10. Ludlow JB, Gubler M, Cevidanes L, Mol A. Precision of cephalometric landmark identification: cone-beam computed tomography vs conventional cephalometric views. Am J Orthod Dentofacial Orthop. 2009;136(3):312.e311-310; discussion 312313.

11. Naji P, Alsufyani NA, Lagravere MO. Reliability of anatomic structures as landmarks in three-dimensional cephalometric analysis using CBCT. Angle Orthod. 2014;84(5):762-772.

12. Olmez H, Gorgulu S, Akin E, Bengi AO, Tekdemir I, Ors F. Measurement accuracy of a computer-assisted three-dimensional analysis and a conventional two-dimensional method. Angle Orthod. 2011;81(3):375-382.

13. de Oliveira AE, Cevidanes LH, Phillips C, Motta A, Burke B, Tyndall D. Observer reliability of three-dimensional cephalometric landmark identification on cone-beam computerized tomography. Oral surgery, oral medicine, oral pathology, oral radiology, and endodontics. 2009;107(2):256-265.

14. Alsufyani NA, Flores-Mir C, Major PW. Three-dimensional segmentation of the upper airway using cone beam CT: a systematic review. Dento maxillo facial radiology. 2012;41(4):276-284.

15. Trpkova B, Major P, Prasad N, Nebbe B. Cephalometric landmarks identification and reproducibility: a meta analysis. Am J Orthod Dentofacial Orthop. 1997;112(2):165170.

16. Lagravere MO, Low C, Flores-Mir C, et al. Intraexaminer and interexaminer reliabilities of landmark identification on digitized lateral cephalograms and formatted 3-dimensional cone-beam computerized tomography images. Am J Orthod Dentofacial Orthop. 2010;137(5):598-604.

17. Schlicher W, Nielsen I, Huang JC, Maki K, Hatcher DC, Miller AJ. Consistency and precision of landmark identification in three-dimensional cone beam computed tomography scans. Eur J Orthod. 2012;34(3):263-275.

18. Sam A, Currie K, Oh H, Flores-Mir C, Lagravere-Vich M. Reliability of different threedimensional cephalometric landmarks in cone-beam computed tomography : A systematic review. Angle Orthod. 2018.

19. Moshiri M, Scarfe WC, Hilgers ML, Scheetz JP, Silveira AM, Farman AG. Accuracy of linear measurements from imaging plate and lateral cephalometric images derived 
from cone-beam computed tomography. Am J Orthod Dentofacial Orthop. 2007;132(4):550-560.

20. Hilgers ML, Scarfe WC, Scheetz JP, Farman AG. Accuracy of linear temporomandibular joint measurements with cone beam computed tomography and digital cephalometric radiography. Am J Orthod Dentofacial Orthop. 2005;128(6):803811.

21. Kosalagood $P$, Silkosessak OC, Pittayapat $P$, Pisarnturakit $P$, Pauwels $R$, Jacobs $R$. Linear Measurement Accuracy of Eight Cone Beam Computed Tomography Scanners. Clinical implant dentistry and related research. 2015;17(6):1217-1227.

22. Park CW, Kim JH, Seo YK, et al. Volumetric accuracy of cone-beam computed tomography. Imaging science in dentistry. 2017;47(3):165-174.

23. Green MN, Bloom JM, Kulbersh R. A simple and accurate craniofacial midsagittal plane definition. Am J Orthod Dentofacial Orthop. 2017;152(3):355-363.

24. Proffit WRT, T. A.;. Dentofacial asymmetry. In: Proffit WRWJ, R. P.;, ed. Surgical orthodontic treatment. St. Louis: Mosby; 1991:483-549.

25. Severt TR, Proffit WR. The prevalence of facial asymmetry in the dentofacial deformities population at the University of North Carolina. The International journal of adult orthodontics and orthognathic surgery. 1997;12(3):171-176.

26. Pittayapat $\mathrm{P}$, Jacobs $\mathrm{R}$, Bornstein $\mathrm{MM}$, et al. Three-dimensional Frankfort horizontal plane for 3D cephalometry: a comparative assessment of conventional versus novel landmarks and horizontal planes. Eur J Orthod. 2018;40(3):239-248.

27. Cevidanes LH, Bailey LJ, Tucker GR, Jr., et al. Superimposition of 3D cone-beam CT models of orthognathic surgery patients. Dento maxillo facial radiology. 2005;34(6):369-375.

28. Afrand M, Ling CP, Khosrotehrani S, Flores-Mir C, Lagravere-Vich MO. Anterior cranial-base time-related changes: A systematic review. Am J Orthod Dentofacial Orthop. 2014;146(1):21-32.e26.

29. Lagravere MO, Gordon JM, Guedes IH, et al. Reliability of traditional cephalometric landmarks as seen in three-dimensional analysis in maxillary expansion treatments. Angle Orthod. 2009;79(6):1047-1056.

30. Lagravere MO, Major PW. Proposed reference point for 3-dimensional cephalometric analysis with cone-beam computerized tomography. Am J Orthod Dentofacial Orthop. 2005;128(5):657-660.

31. Di Carlo G, Fernandez Gurani S, Pinholt EM, Cattaneo PM. A new simple threedimensional method to characterize upper airway in orthognathic surgery patient. Dento maxillo facial radiology. 2017;46(8):20170042. 
32. Donatelli RE, Lee SJ. How to report reliability in orthodontic research: Part 2. Am J Orthod Dentofacial Orthop. 2013;144(2):315-318.

33. Springate SD. The effect of sample size and bias on the reliability of estimates of error: a comparative study of Dahlberg's formula. Eur J Orthod. 2012;34(2):158-163.

34. Molen AD. Considerations in the use of cone-beam computed tomography for buccal bone measurements. Am J Orthod Dentofacial Orthop. 2010;137(4 Suppl):S130-135.

35. Baumrind S, Frantz RC. The reliability of head film measurements. 2. Conventional angular and linear measures. American journal of orthodontics. 1971;60(5):505-517.

36. Pancherz $\mathrm{H}$, Hansen $\mathrm{K}$. The nasion-sella reference line in cephalometry: a methodologic study. American journal of orthodontics. 1984;86(5):427-434.

37. Ford EHR. Growth of the human cranial base. American journal of orthodontics. 1958;44(7):498-506.

38. Melsen B. The cranial base: the postnatal development of the cranial base studied histologically on human autopsy material. P.J. Schmidt, Vojens; 1974.

39. Baumrind S, Miller D, Molthen R. The reliability of head film measurements. 3. Tracing superimposition. American journal of orthodontics. 1976;70(6):617-644.

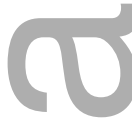

\section{Figure Legends:}

Figure 1: Example of identification of the landmark Nasion in the 3 planes of space. The coronal, axial, sagittal view are displayed together with the 3D rendering.

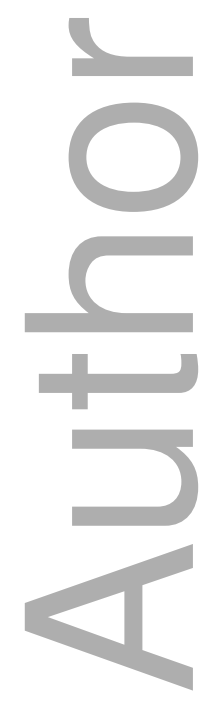



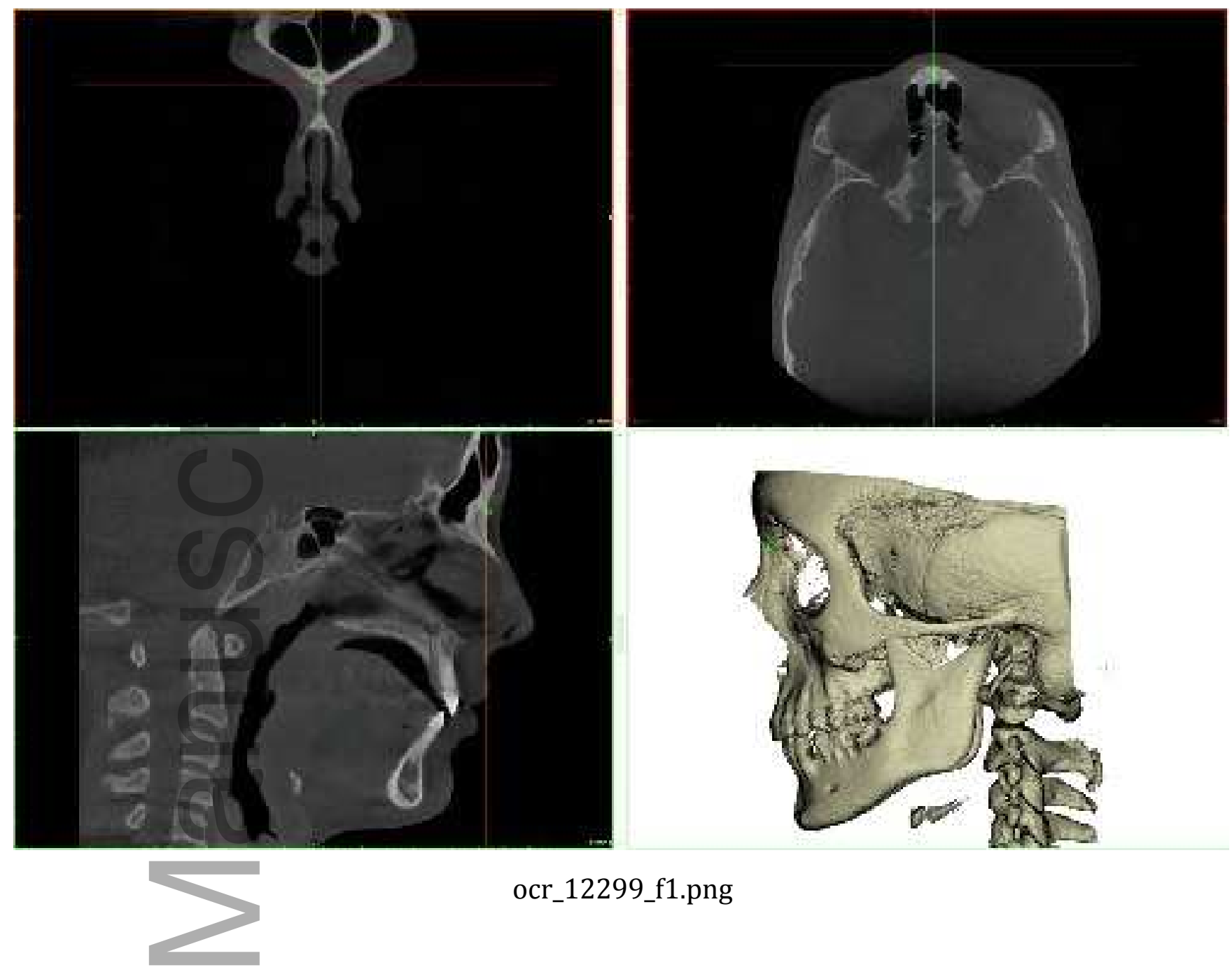

ocr_12299_f1.png

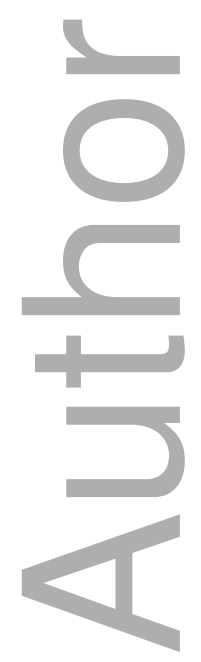

This article is protected by copyright. All rights reserved 


\section{University Library}

\section{- M M N E R VA A gateway to Melbourne's research publications}

Minerva Access is the Institutional Repository of The University of Melbourne

Author/s:

Cattaneo, PM;Yung, AKC;Holm, A;Mashaly, OM;Cornelis, MA

Title:

3D landmarks of Craniofacial Imaging and subsequent considerations on superimpositions in orthodontics-The Aarhus perspective

Date:

2019-05-01

Citation:

Cattaneo, P. M., Yung, A. K. C., Holm, A., Mashaly, O. M. \& Cornelis, M. A. (2019). 3D landmarks of Craniofacial Imaging and subsequent considerations on superimpositions in orthodontics-The Aarhus perspective. ORTHODONTICS \& CRANIOFACIAL RESEARCH, 22 (S1), pp.21-29. https://doi.org/10.1111/ocr.12299.

Persistent Link:

http://hdl.handle.net/11343/285831 